\title{
The spatial control of Torso RTK activation: a C-terminal fragment of the Trunk protein acts as a signal for Torso receptor in the Drosophila embryo
}

\author{
Andreu Casali* and Jordi Casanova \\ Institut de Biologia Molecular de Barcelona (CSIC), C/ Jordi Girona 18-26, 08034 Barcelona, Spain \\ *Present address: Department of Genetics and Development, Columbia University, 701 W 168th street, HHSC 1110 New York, NY 10032, USA \\ ¥Author for correspondence \\ Accepted 19 February; published on WWW 5 April 2001
}

\section{SUMMARY}

Regulated activation of receptor tyrosine kinases depends on both the presence of the receptors at the cell surface and on the availability of their ligands. In Drosophila, the torso tyrosine kinase receptor is distributed along the surface of the embryo but it is only activated at the poles by a diffusible extracellular ligand generated at each pole that is trapped by the receptor, thereby impeding further diffusion. Although it is known that this signal depends on the activity of several genes, such as torso-like and trunk, it is still unclear how is generated. The identification of the signal responsible for the torso receptor activation is an essential step towards understanding the mechanism that regulates the local restriction of torso signalling. Here we report that a fragment containing the carboxy-terminal 108 amino acids of the trunk protein retains trunk activity and is sufficient to activate torso signalling. We also show that this fragment bypasses the requirements for the other genes involved in the activation of the torso receptor. These results suggest that a cleaved form of the trunk protein acts as a signal for the torso receptor. We therefore propose that the restricted activation of the torso receptor is defined by the spatial control of the proteolytic processing of the trunk protein.

Key words: trunk, torso, Signal transduction, Extracellular ligand, Drosophila melanogaster

\section{INTRODUCTION}

Receptor tyrosine kinases are involved in many signalling pathways in cell proliferation and differentiation. Activation of these pathways depends on both the presence of the receptors at the cell surface and on the availability of their ligands. Thus, the extent of receptor activation can be regulated by the limited production or by the restricted diffusion of their ligands (see Jessell and Melton, 1992 for a review). The torso pathway in Drosophila is a particularly appropriate model to address this issue. The torso (Tor) receptor is distributed along all the surface of the Drosophila embryo but it is only activated at the poles, where it specifies development of terminal structures (Schüpbach and Wieschaus, 1986a; Klinger et al., 1988; Casanova and Struhl, 1989; Sprenger et al., 1989). Activation of the Tor receptor has been shown to depend on a diffusible extracellular ligand generated at each pole that is trapped by the receptor, thereby impeding further diffusion (Sprenger and Nüsslein-Volhard, 1992; Casanova and Struhl, 1993).

However, it is still not known which is the terminal signal and how it is generated. torso-like (tsl) and trunk (trk) are two genes that are normally required for activation of the Tor receptor and have been implicated in the local production or action of the Tor ligand (Casanova and Struhl, 1989; Stevens et al., 1990). tsl expression is restricted to specialised follicle cells at each end of the maturing oocyte (Savant-Bhonsale and Montell, 1993; Martin et al., 1994) and its product appears to be found at both embryonic poles after fertilisation (Martin et al., 1994). Moreover, its restricted expression in these follicle cells appears to be critical to generate the localised terminal signal in the embryo because ectopic tsl expression during oogenesis causes central portions of the early embryo to develop terminal structures (Savant-Bhonsale and Montell, 1993; Martin et al., 1994; Furriols et al., 1998). By contrast, trk is required in the female germline (Schüpbach and Wieschaus, 1986b), its RNA is distributed uniformly in the oocyte and codes for a protein with similarities to several types of extracellular growth factors that is likely to be secreted and cleaved (Casanova et al., 1995).

In addition, two other genes, $f s(1)$ pole hole $(f s(1) p h$; also known as female sterile(1) M3) and $f s(1)$ Nasrat $(f s(1) N)$, seem also to be required for local activation of the Tor receptor (Casanova and Struhl, 1989). These genes are special because the majority of mutant alleles at both loci lead to a collapsed egg phenotype probably due to abnormalities in the vitelline membrane surrounding the oocyte and only a particular hypomorphic mutation for each gene gives rise to a terminal phenotype (Degelmann et al., 1990). Thus, it has been proposed that these two genes may have a dual function; they may serve to stabilise the vitelline membrane and they may also be involved in retention or stabilisation of the $t s l$ product in the perivitelline space at the egg poles (Degelmann et al., 1990).

Two models have been suggested for the activation of the 
Tor receptor. In one model, Tsl itself would be the Tor ligand: release of Tsl from its terminal location, perhaps in the vitelline membrane, would activate the Tor receptor in the blastoderm membrane at both poles of the embryo (Martin et al., 1994; see Duffy and Perrimon, 1994 for a review). In another model, Trk might be secreted intact into the perivitelline fluid layer and locally activated by proteolytic cleavage to generate the ligand for the Tor receptor (Casanova et al., 1995). This possibility has been reinforced by the similarity between Trk and spätzle (Spz), the putative ligand for the Toll receptor involved in dorsoventral patterning (Morisato and Anderson, 1994; Schneider et al., 1994). In the first model, restricted activation of Tor signalling would be regulated by the localised deposition of its ligand. In the alternative model, the local processing of a uniformly distributed ligand precursor would regulate the domain of activation of the Tor pathway.

The identification of the signal responsible for Tor receptor activation is an essential step towards understanding the mechanism that regulates the local restriction of Tor activation. Here we report that a fragment containing the carboxy-terminal 108 amino acids of the Trk protein retains Trk activity and is sufficient to activate Tor signalling. We also show that this fragment bypasses the requirements for the other genes involved in the activation of the Tor receptor. These results suggest that a cleaved form of the Trk protein acts as a signal for the Tor receptor. We therefore propose that the restricted activation of the Tor receptor is defined by the spatial control of the proteolytic processing of Trk.

\section{MATERIALS AND METHODS}

\section{Fly strains}

$t_{r k} k^{V 67-1}$ is a mutation that results from the insertion of a $\mathrm{P}$ element and behaves as a null allele (Casanova et al., 1995). tor $^{X R I}$ is a small deficiency that deletes the tor gene (Sprenger et al., 1989). $t s l^{604}$ is a $\mathrm{P}$ element induced mutation (Martin et al., 1994). $f_{s}(1) p h^{1901}$ and $f_{s}(1) N^{211}$ are EMS induced mutations in those genes displaying the specific terminal phenotype (Degelmann et al., 1990). For the ubiquitous early expression of $t r k^{C-108}$, we used the nos-GAL4-VP16 line (Van Doren et al., 1998).

\section{Plasmid constructs}

Full-length trk (pNB-R3)

To introduce the $5^{\prime}$ UTR region of the $\beta$-globin gene in front of a full trk cDNA we cloned a full trk cDNA from position 1987 to 2752 in the genomic map (Casanova et al., 1995) into a pNB40 derivative (Brown and Kafatos, 1988). To generate truncated forms of Trk that would be appropriately secreted we decided to use well-defined sequences that have already been proved to functionally work as signal peptides, even in the context of heterologous proteins (see, for example, Smith and DeLotto, 1994).

trkc-159 $^{c-159}$

We first generated a pea-R3 plasmid by cloning the cDNA of easter (ea) (Chasan and Anderson, 1989) in front of the trk cDNA. By reverse PCR with primers GCCCGCCGATGATTTCGC and TCGTCGTACGAGCTGCCC we generated a plasmid coding for the $e a$ signal peptide up to the glycine residue at position 20 fused to the serine residue of $t r k$ at position 68 .

trk ${ }^{c-108}$

By reverse PCR from pea-R3 with primers GCCCGCCGATGATTTCGC and GTGGGACATCCGAGAATC we generated a plasmid coding for the $e a$ signal peptide up to the glycine residue at position 20 fused to the valine residue of trk at position 119 .

trk ${ }^{c-100}$

We first generated a pwg-R3 plasmid by substituting a trk cDNA fragment from 2025 to 2088 in the genomic map (Casanova et al., $1995)$ by a $93 \mathrm{bp}$ EcoRV-BssHI fragment from wingless $(w g)$ that includes the sequence for its signal peptide (Rijsewijk et al., 1987). By reverse PCR with primers GCCGCTGCACAGGGCCAT and TTTCCACGCTATATCCGTTC we generated a plasmid coding for the $w g$ signal peptide up to the glycine residue at position 17 fused to the phenylalanine residue of $t r k$ at position 148 .

\section{pUAST-trk ${ }^{C-108}$}

The complete coding sequence from the plasmid $t r k^{C-108}$ was excised as an EcoRI-XhoI fragment, cloned in a vector with two NotI flanking sites and inserted as a NotI fragment into the pUAST vector (Brand and Perrimon, 1993). By P-element mediated transformation we generated several independent fly strains carrying the UAS-trk ${ }^{C-108}$ construct.

\section{RNA injections}

RNA was synthesised from $t r k^{C-100}, t^{C-108}$ and $t r k^{C-159}$ plasmids using the mMessage mMachine kit (Ambion) according to the manufacturer's instructions. The RNA was injected at a concentration between 1.5 and $2.5 \mathrm{ng} / \mathrm{nl}$ in the posterior pole of 0 - to 45 -minute-old embryos layed by homozygous mutant females at $17^{\circ} \mathrm{C}$. The embryos were developed for 4 days at $17^{\circ} \mathrm{C}$ and the cuticles were fixed according the standard procedures. Rescue was scored by the presence of the filzkörper.

\section{In situ hybridisation}

$t l l$ and $h k b$ probes were generated from cDNA clones provided by J. Lengyel and H. Jäckle, respectively. Whole-mount in situ hybridisation were done following the method of Tautz and Pfeifle (Tautz and Pfeifle, 1989) with minor modifications.

\section{RESULTS}

\section{Putative cleavage sites in the Trk protein}

The molecular characterisation of the trk gene has shown that it encodes a protein with analogies to extracellular ligands and likely to be cleaved. More precisely, the Trk protein resembles $\mathrm{Spz}$, the putative ligand for the Toll receptor. The analysis of the trk sequence has revealed a putative cleavage site at position 67 in the predicted protein that would generate a carboxy-terminal fragment of 159 amino acids $\left(t r k^{C-159}\right)$. In addition, the conserved arginine immediately upstream of this putative cleavage site is changed to a glutamine in the $t r k^{6}$ lossof-function mutation (Casanova et al., 1995). The observation that a mutation in a putative cleavage site eliminates the biological function of the Trk protein suggests that cleavage is an essential step for Trk activity.

Further analysis of the trk sequence has unveiled an additional putative cleavage site at position 119 , which would give rise to a carboxy-terminal fragment of 108 amino acids $\left(\operatorname{trk}{ }^{C-108}\right)$. Several reasons suggested that this additional site could also be important for Trk activity. First, Trk shares some sequence similarity with the prothoracicotropic hormone (PTTH) of Bombyx mori (data not shown), which is presumed to be cleaved at different sites to yield a final carboxy-terminal fragment of 109 amino acids (Kawakami et al., 1990). Second, Spz is cleaved at a similar position between Arg220 and Val221 
to yield an active fragment of 106 amino acids (Morisato et al., 1994, DeLotto and DeLotto, 1998). The cleavage site of Spz, VSSRVGGS, bears some similarity to the putative cleavage site of Trk, FHDRVGHP (Fig. 1A). And third, most of the active forms of the growth factors with a cystine knot motif possess only a small fragment amino terminal to the first cysteine of the knot (for a review, see McDonald and Hendrickson, 1993). In this regard, the Trk carboxy-terminal fragment generated by cleavage at position $119\left(t^{C} k^{C-108}\right)$ fits this 'rule' better than the fragment generated by cleavage at position 67 (trk $\left.{ }^{C-159}\right)$.

The analysis of the trk sequence, the presence of putative cleavage sites and the mutation on a cleavage site that abolishes trk function prompted us to study the biological activity of deleted forms of the trk gene.

\section{The C-terminal fragment of the Trk protein retains Trk activity}

We have constructed a series of deletions of the trk gene to generate Trk proteins that would lack different amino-terminal fragments while retaining a signal peptide to direct these deleted proteins to the perivitelline space. To generate truncated forms of Trk that would be appropriately secreted we decided to use sequences that had already been characterised as well-defined signal peptides. To assess the activity of these truncated forms, we injected the different transcripts at the posterior pole of embryos laid by trk mutant females. First, as a control, we have verified that trk full-length transcripts were able to rescue the phenotype of embryos from trk mutant females (35\% of rescued embryos; $n=72$ ); we did not detect any rescue when these transcripts were injected in embryos from $t s l$ mutant females (data not shown).

In particular, we have generated two deleted forms that mimic the result of cleavage events at the putative sites in positions 67 and 119. These two forms, $\operatorname{Trk}^{\mathrm{C}-159}$ and $\operatorname{Trk}^{\mathrm{C}-108}$, retain the 159 and the 108 carboxy-terminal residues respectively. We have also generated a third deleted form that retains only the last 100 amino acids, $\operatorname{Trk}^{\mathrm{C}-100}$ (Fig. 1B). Although injection of these truncated transcripts was not very effective, we have found that $\operatorname{Trk}^{\mathrm{C}-159}$ and $\operatorname{Trk}^{\mathrm{C}-108}$ retained trk activity as they were able to partially rescue the trk mutant phenotype as indicated by the appearance of filzkörpers, structures that are never present in trk mutant embryos (Fig. 2 and Table 1). The low percentage of rescued embryos

Table 1. Induction of terminal structures by $t r k^{C-108}$ and trk $^{C-159}$ RNA injection

\begin{tabular}{|c|c|c|c|}
\hline RNA injected & Maternal genotype & Embryos scored & $\mathrm{Fz}(\%)$ \\
\hline \multirow[t]{5}{*}{$\operatorname{trk}^{C-108}$} & $t r k^{V 67-1}$ & 149 & 2 \\
\hline & $t s l^{604}$ & 83 & 9.6 \\
\hline & $f s(1) N^{211}$ & 309 & 3.2 \\
\hline & $f s(1) p h^{1901}$ & 82 & 2.4 \\
\hline & tor $X R I$ & 267 & 0 \\
\hline \multirow[t]{4}{*}{$\operatorname{trk}{ }^{C-159}$} & $\operatorname{trk}^{V 67-1}$ & 88 & 1.1 \\
\hline & $t s l^{604}$ & 118 & 4.3 \\
\hline & $f_{s}(1) N^{211}$ & 221 & 7.7 \\
\hline & $f s(1) p h^{1901}$ & 380 & 1.3 \\
\hline
\end{tabular}

RNA transcripts were injected at the posterior pole of the embryos. Embryos were scored for the differentiation of filzkörper structures $(\mathrm{Fz})$ which are not present in the absence of Tor signalling. obtained with these modified trascripts compared to the fulllength transcript probably reflects the low stability of the truncated mRNAs and/or proteins. Conversely, we could not detect any Trk activity associated with the $\operatorname{Trk}^{\mathrm{C}-100}$ fragment. Thus, we conclude that a truncated Trk protein that contains at least its 108 carboxy-terminal amino acids retains Trk activity.

\section{The C-terminal fragment of Trk bypasses the requirement for the other genes acting in Tor receptor activation}

The ability of $\mathrm{Trk}^{\mathrm{C}-108}$ to induce terminal structures suggested that a carboxy-terminal fragment of the Trk protein could function as an active signal for the Tor receptor once cleaved from its amino-terminus. This raised the possibility that the other genes required for normal activation of the Tor receptor would be involved in the local processing of the Trk protein at the embryonic poles. Consistent with this hypothesis, injections of $t r k^{C-108}$ RNA were also able to induce terminal structures in embryos mutant for $t s l, f s(1) N$ and $f s(1) p h$ (Fig. 2). These experiments indicate that $\operatorname{Trk}^{\mathrm{C}-108}$ bypasses the requirement for these three genes and places trk downstream of $t s l, f s(1) N$ and $f s(1) p h$ in the genetic pathway that controls Tor activation. As expected if $\mathrm{Trk}^{\mathrm{C}-108}$ were activating the Tor receptor, no rescuing activity was observed upon injection in embryos laid by tor mutant females (Fig. 2).

As mentioned above and like $t r k^{C-108}, t^{C} k^{C-159}$ can also induce terminal structures when injected into trk mutant

A

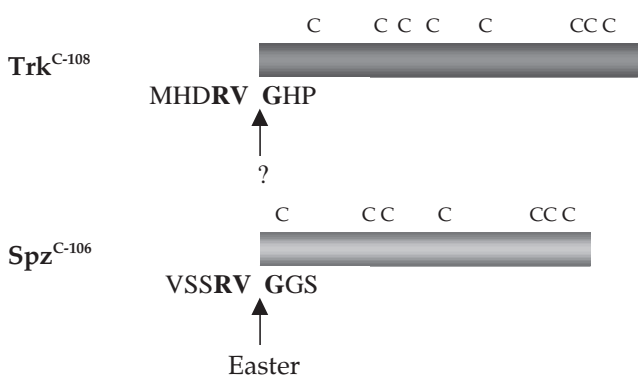

B

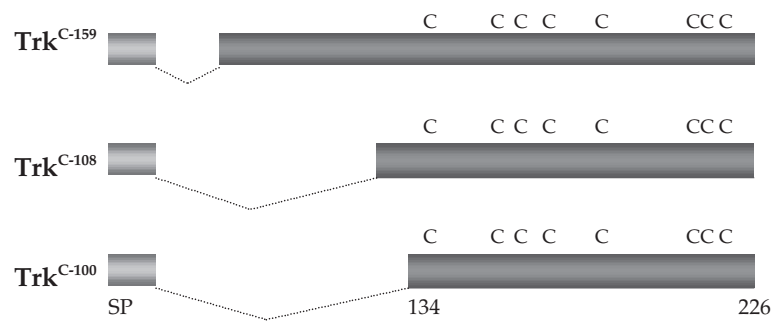

Fig. 1. Generation of deletions in the trk coding region.

(A) Schematic representation of the Spz cleavage and the Trk putative cleavage site at position 119. (B) Schematic representation of the different truncated forms of Trk, $\operatorname{Trk}^{\mathrm{C}-159}, \mathrm{Trk}^{\mathrm{C}-108}$ and $\mathrm{Trk}^{\mathrm{C}-100}$. The light grey box represents the $\mathrm{N}$-terminal signal sequence, from $e a$ in the case of $\mathrm{Trk}^{\mathrm{C}-159}$ and $\mathrm{Trk}^{\mathrm{C}-108}$ and from $w g$ in $\mathrm{Trk}^{\mathrm{C}-100}$. Each $\mathrm{C}$ denotes a cysteine residue. 
Fig. 2. $\operatorname{trk}^{C-108}$ retains Trk activity and bypasses the requirement for the other genes acting in Tor receptor activation. (A) Wild-type embryo. (B) Embryo from a $t^{V 667-1}$ female. Observe that the structures posterior to the seventh abdominal segment are absent. (CF) Embryos from $t r k{ }^{V 67-1}(\mathrm{C}), t s l^{604}$ (D), $f_{s}(1) N^{211}(\mathrm{D})$ and tor ${ }^{X R 1}$

(F) females injected at the posterior pole with $t r k^{C-108}$ RNA. Note the presence of filzkörpers (arrows) in injected embryos from $t r k, t s l$ and $f s(1) N$ mutant females, but not in injected embryos from tor mutant females.
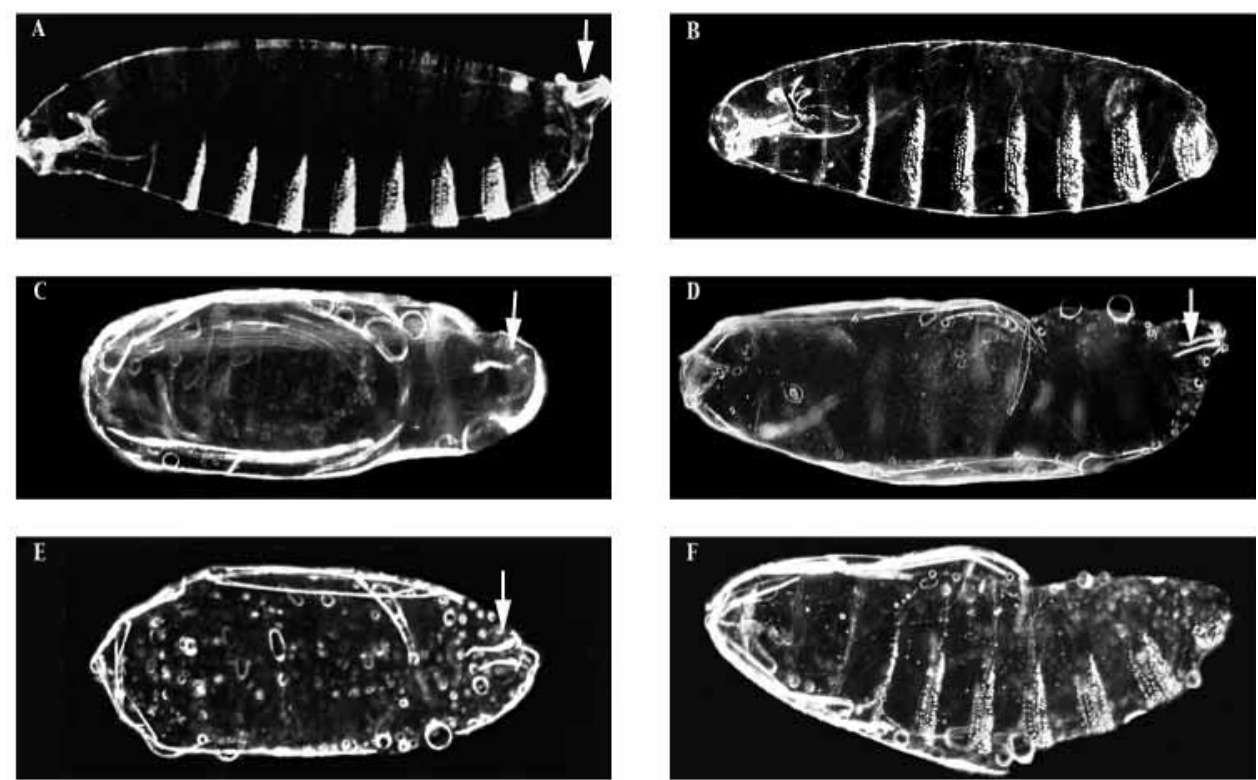

embryos. We therefore addressed the question of whether this larger fragment would behave similarly to the full-length Trk protein and still require the activity of the genes upstream of trk in the genetic pathway. Thus, we assayed the $t r k^{C-159}$ RNA in embryos mutant for $t s l, f s(1) N$ and $f s(1) p h$ and we found that it can also induce terminal structures in these mutant backgrounds (Table 1). We therefore conclude that activity of $\operatorname{Trk}^{\mathrm{C}-159}$ does not depend on any of these genes. Since $\operatorname{Trk}^{\mathrm{C}-108}$ holds the same activity as $\operatorname{Trk}^{\mathrm{C}-159}$, it is possible that $\mathrm{Trk}^{\mathrm{C}-159}$ could represent an intermediate in the generation of an active form of Trk (see Discussion).

\section{The C-terminal fragment of Trk can induce $t / l$ and hkb expression}

The activity of the deleted forms of the trk gene upon mRNA injection was assessed by the appearance of filzkörpers in the cuticle of the developed embryos. In this assay, few embryos could be scored as positive. As mentioned above, the efficiency of the injections was very poor probably due to the low stability of the truncated mRNAs and/or proteins. Thus, it was crucial to analyse whether the C-terminal fragment of Trk could trigger all the downstream responses of the Tor signalling pathway. The zygotic genes tailless (tll) and huckebein $(h k b)$ appear to be the only targets of Tor activation at the posterior pole of the embryo (Weigel et al., 1990). More precisely, only high levels of Tor signalling appear to be able to activate $h k b$ expression (Furriols et al., 1996). Thus, to further characterise the activity of the C-terminal fragment of the Trk protein we have examined whether it could induce $t l l$ and $h k b$ expression in mutant embryos for $t s l$. To overcome the difficulties of the RNA injection assay, we have generated transgenic lines expressing $\operatorname{Trk}^{\mathrm{C}-108}$ under the control of the UAS promoter (Brand and Perrimon, 1993). We used these lines to obtain ubiquitous expression of $\operatorname{Trk}^{\mathrm{C}-108}$ at early embryonic stages by means of a maternal GAL4 line (see Materials and Methods). These conditions were sufficient to provide full terminal activity as they could induce both $t l l$ and $h k b$ expression in embryos mutant for $t s l$ (Fig. 3).
The C-terminal fragment of Trk can induce phenotypes associated with general activation of the Tor receptor

The above experiments indicate that a general level of $\operatorname{Trk}^{\mathrm{C}-108}$ can generate restricted expression of the target genes of the Tor signalling at both poles of the embryo. Similar results are observed when Tor signalling is activated at low levels all over the embryo, either by general expression of $t s l$ (Furriols et al., 1998) or by a constitutive ligand-independent mutation in the Tor receptor (Steingrímsson et al., 1991). Only when higher levels of Tor signalling are achieved it is possible to detect its deleterious effect in the central regions of the embryo. Consistently, we have observed that some embryos derived from females bearing three copies of the UAS-trk ${ }^{C-108}$ and a maternal GAL4 insertion (see Materials and Methods) have deletions of the middle body segments (Fig. 4). This is the phenotype associated with the expansion of the terminal portions of the embryo at the expense of the central region (Klinger et al., 1988), a phenotype that is more sensitive to weak general activation of the Tor receptor than in situ analysis for $t l l$ and $h k b$ expression (see for example, Cleghon et al., 1996 and Furriols et al., 1998). This result suggests that the C108 carboxy-terminal fragment of Trk is sufficient to induce terminal fates all over the embryo.

\section{DISCUSSION}

\section{A C-terminal fragment of Trk can act as a signal for the Tor receptor}

The molecular characterisation of the trk gene suggested that it could encode the ligand for the Tor receptor. In particular, this possibility was sustained by the finding that the Trk protein might be secreted and cleaved to yield a carboxy-terminal polypeptide. This carboxy-terminal fragment displays an arrangement of cysteines reminiscent of that of the cystine knot motif present in several growth factors. Specifically, the similarities between Trk and Spz, which is thought to be the ligand for the Toll receptor in the establishment of the 
Fig. 3. $\operatorname{Trk}^{\mathrm{C}-108}$ can induce $t l l$ and $h k b$ expression. (A-B) In wild-type embryos, $t l l$ (A) and $h k b$ (B) expression is restricted to the poles. (C-D) In embryos from $t s^{l 604}$ females, $t l l(\mathrm{C})$ and $h k b$ (D) expression is missing at the posterior pole; expression remains in the anterior domain due to the activity of the anterior system. (E-F) Uniform expression of $t r k^{C-108}$ by a nos-GAL4-VP16 driver restores the ability of the terminal system to direct the expression of $t l l(\mathrm{E})$ and $h k b(\mathrm{~F})$ at the poles of embryos from $t s 1^{604}$ females.
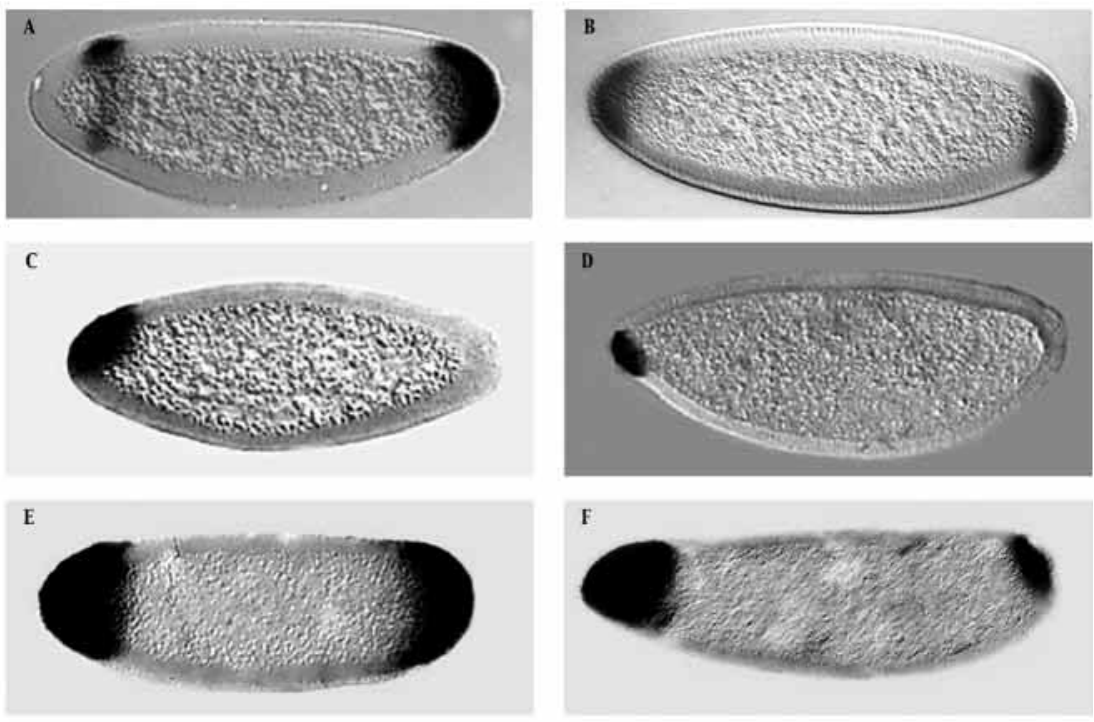

Drosophila dorsoventral axis, raised the possibility that Spz and Trk could function in an analogous fashion (Casanova et al., 1995). However, this notion was not in agreement with previous studies that indicated that the properties of Tsl protein were consistent with its role as the Tor ligand (Martin et al., 1994). Therefore, the nature of the Tor ligand, and hence the mechanism of Tor activation, has remained unresolved. The results reported here clearly substantiate that a C-terminal fragment of the Trk protein can act as a signal for the Tor receptor. Although the injection of the truncated versions of the trk RNA is rather inefficient in terms of the percentage of rescued embryos, our results indicate that a C-terminal fragment of Trk is sufficient to trigger all the known responses of the Tor signalling pathway. In particular, the C-terminal fragment of Trk is able to induce these responses in the absence of the function of the other genes required for Tor receptor activation, including $t s l$. Besides, general expression of a Cterminal fragment of Trk is sufficient to produce similar phenotypes to those associated with the ubiquitous activation of the Tor receptor. These results single out the product of the trk gene as the only one sufficient and absolutely required for the activation of the Tor receptor, even though other gene functions could collaborate in the process.

The C-terminal domain of Trk is sufficient to activate the Tor receptor but its activity appears to be inhibited by the presence of the amino-terminal domain of the protein. Although the deletion mutations that we have generated may not represent the actual in vivo protein, we favour the hypothesis that the truncated proteins could mimic the in vivo active protein and relieve the C-terminal region from such an inhibition. In particular, the observation that a mutation in a putative cleavage site eliminates the biological function of the Trk protein (Casanova et al., 1995) suggests that cleavage is physiologically relevant for Trk activity and weakens the possibility that the designed truncated forms would artificially alter the normal process of Trk protein activation.

\section{A two step processing of the Trk protein?}

The analysis of trk has unveiled the existence of two putative cleavage sites. Indeed, we have found that two different deletions generated at each site that retain 159 and 108 amino acids respectively are both able to activate Tor signalling in the embryos upon RNA injection. Although we have already mentioned that these two mutant forms may not represent the actual in vivo active protein, we favour the hypothesis that the smaller fragment could be similar to the in vivo active Trk fragment whereas the larger one could correspond to an intermediate form. In that case, the in vivo processing of the Trk protein could involve more that one cleavage event. Several observations support this model. Firstly, Trk ${ }^{\mathrm{C}-108}$ is sufficient to activate Tor signalling as indicated by the expression of both $t l l$ and $h k b$, suggesting that the additional amino acids in $\mathrm{Trk}^{\mathrm{C}-158}$ are dispensable for Tor activation. In addition, $\operatorname{Trk}^{\mathrm{C}-108}$ fits very well with the other known active cystine-knot factors, both in its overall size and in the small amino acid chain just before the first cysteine of the knot (see McDonald and Hendrickson, 1993). Finally, as discussed above, the putative cleavage site at the basis of $\operatorname{Trk}^{\mathrm{C}-158}$ appears to be functionally important as a specific mutation at this site renders the protein inactive (Casanova et al., 1995). Thus, both cleavage events could be taking place in the in vivo processing of Trk. A similar situation has been described for another putative cystine-knot protein, the PTTH hormone of Bombyx

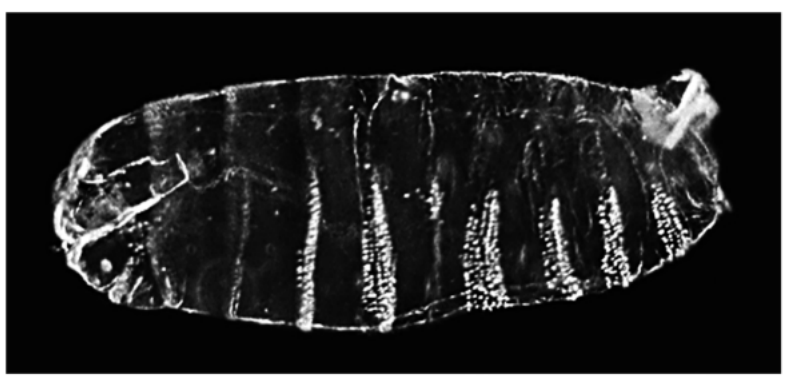

Fig. 4. $\operatorname{trk}^{\mathrm{C}-108}$ can induce deletions of the middle segments of the embryo. Cuticle phenotype of an embryo expressing a large amount of $t r k^{C-108}$ (derived from a female bearing 3 copies of the UAStrk ${ }^{C-108}$ and a nos-GAL4-VP16 insertion). Observe the deletion of the middle embryonic segments, a phenotype typically associated with ectopic activation of the Tor receptor. 


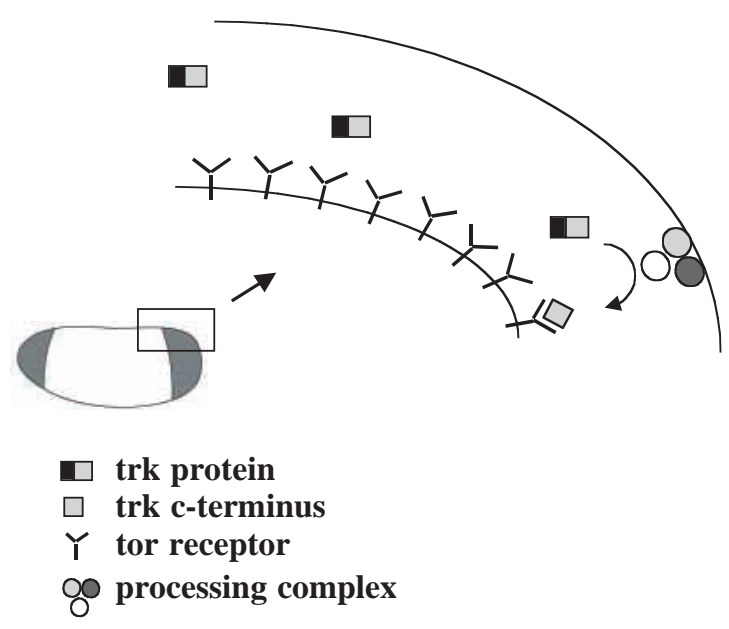

Fig. 5. A model for the mechanism of Tor receptor activation. The Tor receptor is distributed along all the surface of the Drosophila embryo but it is only activated at the poles (shaded regions). Trk is secreted into the perivitelline fluid layer as an inactive ligand and is proteolytically cleaved by a processing complex restricted to the poles. Thus, the active ligand is generated at the poles and trapped by the receptor. In this model, $t s l, f_{s}(1) N$ and $f_{s}(1) p h$ gene products could participate in the formation of the processing complex.

mori (Kawakami et al., 1990), which bears some sequence similarity to Trk (data not shown). Likewise, the Trk active form could arise from the cleavage at position 119 that would require a previous cleavage at position 67 . It has to be noted that in this model the cleavage at position 67 could be the target of the locally restricted processing at the embryonic poles since $\operatorname{Trk}^{\mathrm{C}-158}$ can already bypass the requirement for the other genes needed for local activation of the Tor receptor. Conversely, cleavage at position 119 would not need to be locally controlled.

\section{The spatial control of Tor receptor activation}

The Tor receptor is distributed all along the embryonic surface but it is only activated at the poles by a still unknown mechanism. Two models have been proposed for the locally restricted activation of the Tor receptor. In one model, the Tsl protein that is secreted from a subset of follicular cells at both poles of the oocyte would act as a ligand for the Tor receptor (Martin et al., 1994). In such a scenario, the spatial restriction in the activation of the Tor receptor would lie in the local production of its ligand. In an alternative model, Trk would be secreted into the perivitelline fluid layer and then activated at the embryonic poles by a proteolytic cleavage to generate the ligand for the Tor receptor (Casanova et al., 1995). In this alternative model, based on the characteristics of the Trk protein, the spatial restriction in the activation of the Tor receptor would lie in the local processing of its ligand. Our experiments clearly support this second model and suggest that the other genes required for Tor activation are involved in the local generation of a Trk active form.

The molecular characterisation of the trk gene unveiled the similarities between Trk and Spz, the presumed ligand for the Toll receptor. The present experiments extend the similarity between the terminal and the dorsoventral system as they indicate that in both systems a transmembrane receptor is activated by a ubiquitous ligand that is asymmetrically cleaved into an active form. However, while a well characterised cascade of serine proteases has been identified in the dorsoventral system (Chasan and Anderson, 1989; DeLotto and Spierer, 1986; Hong and Hashimoto, 1995, Konrad et al., 1998), there are no known genes coding for proteases in the terminal system. Since the terminal genes $f s(1) p h$ and $f_{s}(1) N$ have not yet been molecularly characterised it is possible that one of them could code for a protease. In this context it is interesting to note that nudel ( $n d l)$, one of the genes coding for a protease in the dorsoventral system, is also needed for vitelline membrane integrity (Hong and Hashimoto, 1995), a function that is also shared by both $f_{s}(1) p h$ and $f_{s}(1) N$ (Degelmann et al., 1990). Still, it is also possible that additional genes required for Tor receptor activation remain to be identified.

A critical step in both systems is the mechanism that triggers the production of the active form of the ligand. In the dorsoventral system this mechanism is initiated by the restricted expression of pipe in the ventral follicle cells. pipe codes for heparan sulfate 2-O-sulfotransferase that modifies the glycosaminoglycan (GAG) side chain of proteoglycans (Sen et al., 1998). Modification of Ndl, or of a yet unknown substrate, by pipe would in turn allow the nucleation of a protein complex that would begin the proteolytic cascade leading to the production of active Spz. Much less is known about this mechanism in the terminal system, which is also initiated by the restricted expression of a particular gene in a subset of follicle cells. In this case, tsl expression in some follicle cells at each end of the maturing oocyte defines the restricted domain of Tor activation (Savant-Bhonsale and Montell, 1993; Martin et al., 1994). However, it is not clear how $t s l$ activity is translated into the production of an active form of the Tor ligand. The only specific characteristic of the Tsl protein is the presence of leucine-rich repeats which could mediate protein-protein or protein-lipid interactions (SavantBhonsale and Montell, 1993). Thus, it is possible that the Tsl product could constitute the link binding a processing complex to the membrane, but other alternative mechanisms are also possible. The understanding of the mechanism that translates the original asymmetry in the follicle cells to the restricted production of active Trk will require the characterisation of the additional genes needed for Trk processing and thus for Tor activation.

We thank M. Furriols for his contribution in the initiation of this work. We thank A. González-Reyes, H. Jäckle, J. Lengyel, R. Lehmann, A. Mahowald, D. Montell and F. Sprenger for providing flies and materials, and G. Jiménez and M. Furriols for critical comments on the manuscript. We also thank Nicolás Martín for his technical assistance. A. C. was supported by a fellowship from the Ministerio de Educación y Ciencia and from the Marató de TV3. This work was carried out in the framework of the Centre de Referència en Biotecnologia de la Generalitat de Catalunya and supported by the DGICYT.

\section{REFERENCES}

Brand, A. H. and Perrimon, N. (1993). Targeted gene expression as a means of altering cell fates and generating dominant phenotypes. Development, 118, 401-415.

Brown, N. H. and Kafatos, F. C. (1988). Functional cDNA libraries from Drosophila embryos. J. Mol. Biol. 203, 425-437. 
Casanova, J. and Struhl, G. (1989). Localized surface activity of torso, a receptor tyrosine kinase, specifies terminal body pattern in Drosophila. Genes Dev. 3, 2025-2038.

Casanova, J. and Struhl, G. (1993). The torso receptor localizes as well as transduces the spatial signal specifying terminal body pattern in Drosophila. Nature 362, 152-155.

Casanova, J., Furriols, M., McCormick, C. A. and Struhl, G. (1995). Similarities between trunk and spätzle, putative extracellular ligands specifying body pattern in Drosophila. Genes Dev. 9, 2539-2544.

Chasan, R. and Anderson, K. V. (1989). The role of easter, an apparent serine protease, in organizing the dorsal-ventral pattern of the Drosophila embryo. Cell 56, 391-400.

Cleghon, V., Gayko, U., Copeland, T. D., Perkins, L. A., Perrimon, N. and Morrison, D. K. (1996). Drosophila terminal structure development is regulated by the compensatory activities of positive and negative phosphotyrosine signaling sites on the Torso RTK. Genes Dev. 10, 566-577.

Degelmann, A., Hardy, P. A. and Mahowald, A. P. (1990). Genetic analysis of two female-sterile loci affecting eggshell integrity and embryonic pattern formation in Drosophila melanogaster. Genetics 126, 427-434.

DeLotto, R. and Spierer, P. (1986). A gene required for the specification of dorsal-ventral pattern in Drosophila appears to encode a serine protease. Nature 323, 688-692.

DeLotto, Y. and DeLotto, R. (1998). Proteolytic processing of the Drosophila Spätzle protein by Easter generates a dimeric NGF-like molecule with ventralising activity. Mech. Dev. 72, 141-148.

Duffy, J. B. and Perrimon, N. (1994). The Torso pathway in Drosophila: lessons on receptor tyrosine kinase signaling and pattern formation. Dev. Biol. 166, 380-395.

Furriols, M., Casali, A. and Casanova J. (1998). Dissecting the mechanism of torso receptor activation. Mech. Dev. 70, 111-118.

Furriols, M., Sprenger, F. and Casanova J. (1996). Variation in the number of activated Torso receptors correlates with differential gene expression. Development 122, 2313-2317.

Hong, C. C. and Hashimoto, C. (1995). An unusual mosaic protein with a protease domain, encoded by the nudel gene, is involved in defining embryonic dorsoventral polarity in Drosophila. Cell 82, 785-794.

Jessell, T. M. and Melton, D. A. (1992). Diffusible factors in vertebrate embryonic induction. Cell 68, 257-270.

Kawakami, A., Kataoka, H., Oka,T., Mizoguchi, A., Kimura-Kawakami, M., Adachi, T., Iwami, M., Nagasawa, H., Suzuki, A. and Ishizaki, H. (1990). Molecular cloning of the Bombyx mori prothoracicotropic hormone. Science 247, 1333-1335.

Klingler, M., Erdelyi, M., Szabad, J. and Nüsslein-Volhard, C. (1988). Function of torso in determining the terminal anlagen of the Drosophila embryo. Nature 335, 275-277.

Konrad, K. D., Goralski, T. J., Mahowald, A. P. and Marsh, J. L. (1998) The gastrulation defective gene of Drosophila melanogaster is a member of the serine protease superfamily. Proc. Natl. Acad. Sci. USA 95, 68196824.

Martin, J.-R., Raibaud A. and Ollo, R. (1994). Terminal pattern elements in Drosophila embryo induced by the torso-like protein. Nature $\mathbf{3 6 7}, 741$ 745 .

McDonald, N. Q. and Hendrickson, W. A. (1993). A structural superfamily of growth factors containing a cysteine knot motif. Cell 73, 421-424.

Morisato, D. and Anderson, K. V. (1994). The spätzle gene encodes a component of the extracellular signaling pathway establishing the dorsoventral pattern of the Drosophila embryo. Cell 76, 677-688.

Rijsewijk, F. Schuermann, M., Wagenaar, E., Parren, P., Weigel, D. and Nusse, R. (1987). The Drosophila homolog of the mouse mamary oncogene int-1 is identical to segmental polarity gene wingless. Cell 50, 649-657.

Savant-Bhonsale, S. and Montell, D. J. (1993). torso-like encodes the localized determinant of Drosophila terminal pattern formation. Genes Dev. 7, 2548-2555.

Schneider, D. S., Jin, Y., Morisato, D. and Anderson, K. V. (1994). A processed form of Spätzle protein defines dorso-ventral polarity in the Drosophila embryo. Development 120, 1243-1250.

Schüpbach, T. and Wieschaus, E. (1986a). Maternal-effect mutations altering the anterior-posterior pattern of the Drosophila embryo. Roux's Arch. Dev. Biol. 195, 302-317.

Schüpbach, T. and Wieschaus, E. (1986b). Germ-line autonomy of maternaleffect mutations altering the embryonic body pattern of Drosophila. Develop. Biol. 113, 443-338.

Sen, J., Goltz, J. S., Stevens, L. and Stein, D. (1998). Spatially restricted expression of pipe in the Drosophila egg chamber defines embryonic Dorsal-Ventral polarity. Cell 95, 471-481.

Smith, C. L. and and DeLotto, R. (1994). Ventralizing signal determined by protease activation in Drosophila embryogenesis. Nature 368, 548-551.

Sprenger, F., Stevens, L. M. and Nüsslein-Volhard, C. (1989). The Drosophila gene torso encodes a putative receptor tyrosine kinase. Nature 338, 478-483.

Sprenger, F. and Nüsslein-Volhard, C. (1992). Torso receptor activity is regulated by a diffusible ligand produced at the extracellular terminal regions of the Drosophila egg. Cell 71, 987-1001.

Steingrímson, E., Pignoni, F., Liaw, G.-J. and Lengyel, J. A. (1991). Dual role of the Drosophila pattern gene tailless in embryonic termini. Science 254, 418-421.

Stevens, L. M., Fronhöfer, H. G., Klinger, M. and Nüsslein-Volhard, C. (1990). Localized requirement for torso-like expression in follicle cells for development of the terminal anlagen of the Drosophila embryo. Nature 346, 660-663.

Tautz, D. and Pfeifle, C. (1989) A non-radioactive in situ hybridization method for the localization of specific RNAs in Drosophila embryos reveals translational control of the segmentation gene hunchback. Chromosoma, 98, 81-85.

Van Doren, M., Williamson, A. and Lehmann, R. (1998). Regulation of zygotic gene expression in Drosophila primordial germ cells. Curr. Biol. 8, 243-246.

Weigel, D., Jürgens, G., Klinger, M. and Jäckle, H. (1990). Two gap genes mediate maternal terminal pattern information in Drosophila. Science $\mathbf{2 4 8}$, 495-498. 